\title{
Study of the photon strength functions and level density in the gamma decay of the $n+{ }^{234} \mathrm{U}$ reaction
}

J. Moreno-Soto ${ }^{1, *}$, E. Berthoumieux ${ }^{1}$, E. Dupont ${ }^{1}$, F. Gunsing ${ }^{1}$, O. Serot $^{2}$, O. Litaize ${ }^{2}$, M. Diakaki ${ }^{2}$ A. Chebboubi ${ }^{2}$, W. Dridi ${ }^{3}$, S. Valenta ${ }^{33}$, M. Krtička ${ }^{33}$, O. Aberle ${ }^{4}$, V. Alcayne ${ }^{5}$, J. Andrzejewski ${ }^{6}$, L. Audouin ${ }^{7}$, V. Bécares ${ }^{5}$, V. Babiano-Suarez ${ }^{8}$, M. Bacak ${ }^{4,9,1}$, M. Barbagallo ${ }^{4,10}$, Th. Benedikt ${ }^{11}$, S. Bennett ${ }^{12}$, J. Billowes ${ }^{12}$, D. Bosnar ${ }^{13}$, A. Brown ${ }^{14}$, M. Busso ${ }^{10,15,16}$, M. Caamaño ${ }^{17}$, L. Caballero-Ontanaya ${ }^{8}$, F. Calviño ${ }^{18}$, M. Calviani ${ }^{4}$, D. Cano-Ott ${ }^{5}$, A. Casanovas $^{18}$, F. Cerutti ${ }^{4}$, E. Chiaveri ${ }^{4,12}$, N. Colonna ${ }^{10}$, G. Cortés ${ }^{18}$, M. A. Cortés-Giraldo ${ }^{19}$, L. Cosentino ${ }^{20}$, S. Cristallo ${ }^{10,15,21}$, L. A. Damone ${ }^{10,22}$, P. J. Davies ${ }^{12}$, M. Dietz ${ }^{23}$, C. Domingo-Pardo ${ }^{8}$, R. Dressler ${ }^{24}$, Q. Ducasse ${ }^{25}$, I. Durán ${ }^{17}$, Z. Eleme ${ }^{26}$, B. Fernández-Domínguez ${ }^{17}$, A. Ferrari ${ }^{4}$, P. Finocchiaro ${ }^{20}$, V. Furman ${ }^{27}$, K. Göbel ${ }^{11}$, A. Gawlik ${ }^{6}$, S. Gilardoni ${ }^{4}$, I. F. Gonçalves ${ }^{28}$, E. González-Romero ${ }^{5}$, C. Guerrero ${ }^{19}$, S. Heinitz ${ }^{24}$, J. Heyse ${ }^{29}$, D. G. Jenkins ${ }^{14}$, A. Junghans ${ }^{30}$, F. Käppeler ${ }^{31}$, Y. Kadi ${ }^{4}$, A. Kimura ${ }^{32}$, I. Knapova ${ }^{33}$, M. Kokkoris ${ }^{34}$, Y. Kopatch ${ }^{27}$, D. Kurtulgil ${ }^{11}$, I. Ladarescu ${ }^{8}$, C. Lederer-Woods ${ }^{23}$, S. J. Lonsdale ${ }^{23}$, D. Macina ${ }^{4}$, A. Manna ${ }^{35,36}$, T. Martínez ${ }^{5}$, A. Masi ${ }^{4}$, C. Massimi $^{35,36}$, P. Mastinu ${ }^{37}$, M. Mastromarco ${ }^{4}$, E. A. Maugeri ${ }^{24}$, A. Mazzone ${ }^{10,38}$, E. Mendoza $^{5}$, A. Mengoni ${ }^{39}$, V. Michalopoulou ${ }^{4,34}$, P. M. Milazzo ${ }^{40}$, F. Mingrone ${ }^{4}$, A. Musumarra ${ }^{20,41}$, A. Negret ${ }^{42}$, F. Ogállar ${ }^{43}$, A. Oprea ${ }^{42}$, N. Patronis ${ }^{26}$, A. Pavlik ${ }^{44}$, J. Perkowski ${ }^{6}$, L. Persanti ${ }^{10,15,21}$, C. Petrone ${ }^{42}$, E. Pirovano ${ }^{25}$, I. Porras ${ }^{43}$, J. Praena ${ }^{43}$, J. M. Quesada ${ }^{19}$, D. Ramos-Doval ${ }^{7}$, T. Rauscher ${ }^{45,46}$, R. Reifarth ${ }^{11}$, D. Rochman ${ }^{24}$, M. Sabaté-Gilarte ${ }^{19,4}$, A. Saxena ${ }^{47}$, P. Schillebeeckx ${ }^{29}$, D. Schumann ${ }^{24}$, A. Sekhar ${ }^{12}$, S. Simone ${ }^{20}$, A. G. Smith ${ }^{12}$, N. V. Sosnin ${ }^{12}$, P. Sprung ${ }^{24}$, A. Stamatopoulos ${ }^{34}$, G. Tagliente ${ }^{10}$, J. L. Tain ${ }^{8}$, A. Tarifeño-Saldivia ${ }^{18}$, L. Tassan-Got ${ }^{4,34,7}$, A. Tsinganis ${ }^{4}$, J. Ulrich ${ }^{24}$, S. Urlass ${ }^{30,4}$, G. Vannini ${ }^{35,36}$, V. Variale ${ }^{10}$, P. Vaz $^{28}$, A. Ventura ${ }^{35}$, D. Vescovi ${ }^{10,15}$, V. Vlachoudis ${ }^{4}$, R. Vlastou ${ }^{34}$, A. Wallner ${ }^{48}$, P. J. Woods ${ }^{23}$, T. Wright ${ }^{12}$, P. Žugec ${ }^{13}$, and the n_TOF Collaboration

${ }^{1}$ CEA Irfu, Université Paris-Saclay, F-91191 Gif-sur-Yvette, France

${ }^{2}$ CEA, DEN, DER, SPRC, Cadarache, F-13108 Saint-Paul-lez-Durance, France

${ }^{3}$ Laboratory on Energy and Matter for Nuclear Sciences Development (LR16CNSTN02), Technopark Sidi Thabet, 2020 Ariana, Tunisia

${ }^{4}$ European Organization for Nuclear Research (CERN), Switzerland

${ }^{5}$ Centro de Investigaciones Energéticas Medioambientales y Tecnológicas (CIEMAT), Spain

${ }^{6}$ University of Lodz, Poland

${ }^{7}$ Institut de Physique Nucléaire, CNRS-IN2P3, Univ. Paris-Sud, Université Paris-Saclay, F-91406 Orsay Cedex, France

${ }^{8}$ Instituto de Física Corpuscular, CSIC - Universidad de Valencia, Spain

${ }^{9}$ Technische Universität Wien, Austria

${ }^{10}$ Istituto Nazionale di Fisica Nucleare, Sezione di Bari, Italy

${ }^{11}$ Goethe University Frankfurt, Germany

${ }^{12}$ University of Manchester, United Kingdom

${ }^{13}$ Department of Physics, Faculty of Science, University of Zagreb, Zagreb, Croatia

*e-mail: javier.moreno-soto@cea.fr 


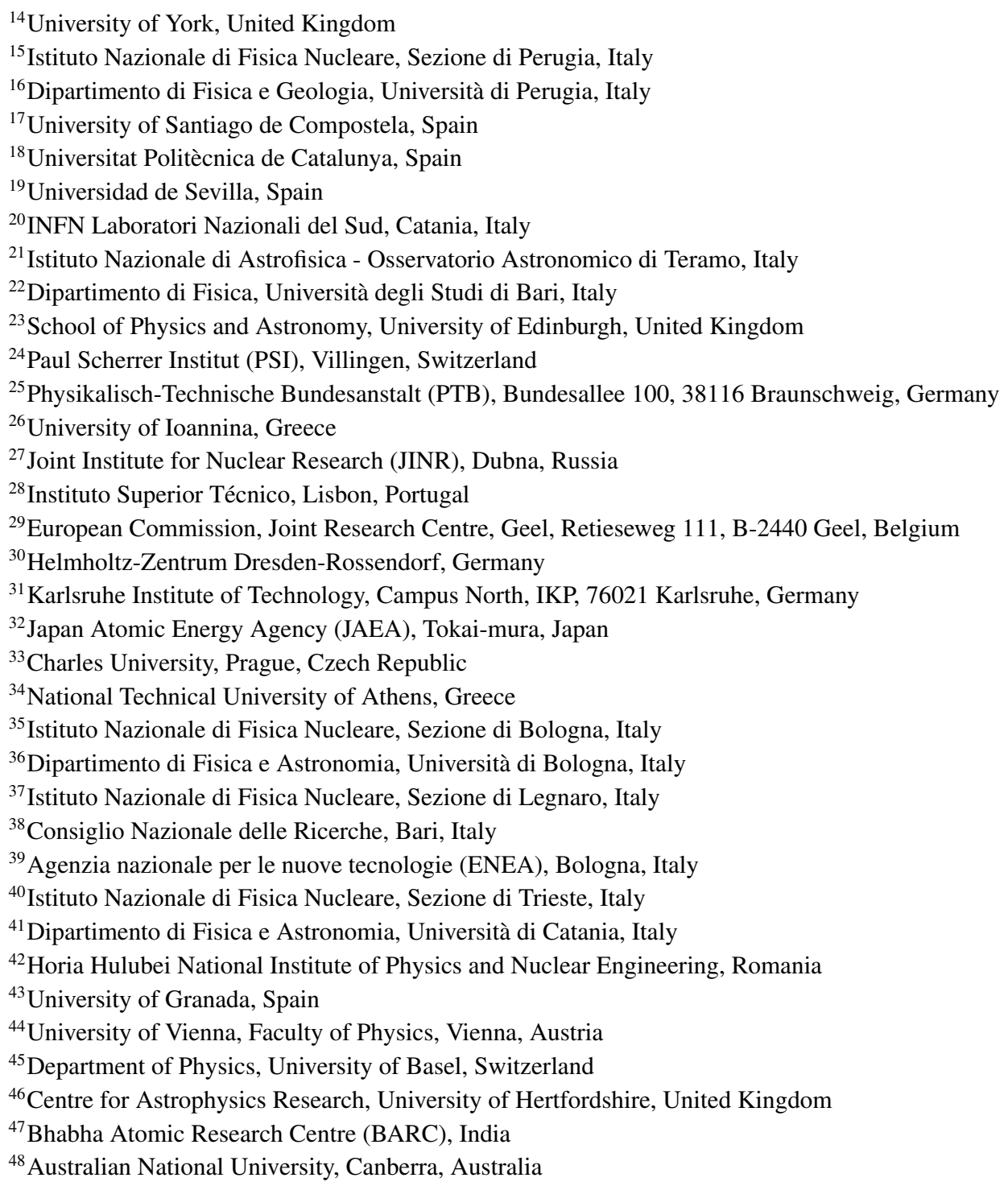

Abstract. The accurate calculations of neutron-induced reaction cross sections are relevant for many nuclear applications. The photon strength functions and nuclear level densities are essential inputs for such calculations. These quantities for ${ }^{235} \mathrm{U}$ are studied using the measurement of the gamma de-excitation cascades in radiative capture on ${ }^{234} \mathrm{U}$ with the Total Absorption Calorimeter at n_TOF at CERN. This segmented $4 \pi$ gamma calorimeter is designed to detect gamma rays emitted from the nucleus with high efficiency. This experiment provides information on gamma multiplicity and gamma spectra that can be compared with numerical simulations. The code DicEвoxc is used to simulate the gamma cascades while GEANT4 is used for the simulation of the interaction of these gammas with the TAC materials. Available models and their parameters are being tested using the present data. Some preliminary results of this ongoing study are presented and discussed. 


\section{Introduction}

The aim of this work is to describe the $\gamma$-decay of excited nucleus following neutron capture. At low excitation energy, the number of levels per unit energy is rather small and levels can be experimentally resolved. However, as the excitation energy increases the level density is also increasing, so the statistical model is needed to describe the levels and transitions between them - the used quantities are nuclear level density (LD) and photon strength functions (PSFs). Their improved experimental and theoretical description is important for modeling of radiative capture reactions in nuclear astrophysics and nuclear technologies since the neutron capture cross sections above the resolved resonance region are usually calculated using the statistical model of Hauser-Feshbach [1] for which PSFs and LDs are essential inputs.

In this work, the Total Absorption Calorimeter (TAC) at n_TOF facility (CERN) [2-4] was used to measure ${ }^{234} \mathrm{U}(\mathrm{n}, \gamma)$ reaction [5]. The TAC is a $4 \pi$ detector segmented in $40 \mathrm{BaF}_{2}$ crystals with a very high efficiency (almost 100\%) to detect the $\gamma$ rays from the capture cascades. In Fig. 1 (left) one hemisphere of the TAC is shown. The ${ }^{234} \mathrm{U}$ sample is placed in the center and emits $\gamma$ rays, which are detected by the $\mathrm{BaF}_{2}$ detectors. Thanks to the segmentation of the detector it is possible to discriminate against the background by putting conditions on the multiplicity and the total deposited energy of events registered by the TAC.
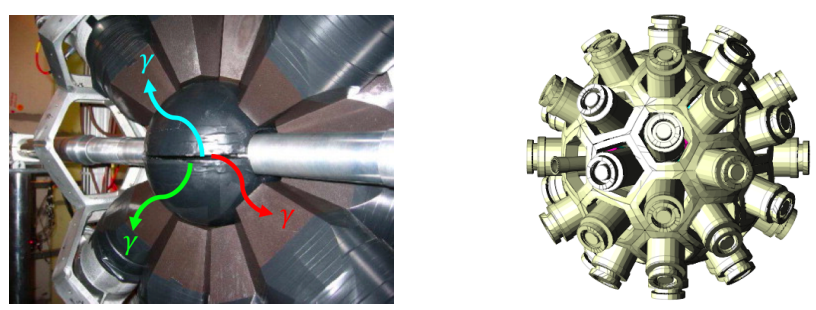

Figure 1. One hemisphere of the TAC consisting of the $\mathrm{BaF}_{2}$ detectors, the neutron beam tube and the neutron absorber. A cascade event of three $\gamma$ rays is depicted. If all $\gamma$ rays are detected the crystal multiplicity is $m_{c r}=3$ (left). The geometry of the full TAC as implemented in GEANT4 (right).

\section{Experimental data}

In a radiative capture reaction the compound nucleus decays through a cascade of $\gamma$ rays. The measured cascade events are reconstructed by taking $\gamma$ rays detected by the TAC in the $\mathrm{BaF}_{2}$ detectors in a time coincidence window of $20 \mathrm{~ns}$. A software threshold of $75 \mathrm{keV}$ was set for all $\mathrm{BaF}_{2}$ detectors to suppress the low energy background. The observables used for the analysis are:

- The neutron energy, $E_{n}$, calculated from the measured time of flight.

- The crystal multiplicity, $m_{c r}$, given by the number of hit crystals in each detected cascade event.

- The total deposited energy or sum energy $E_{\text {sum }}$ in the detectors for each cascade event.

- The multi-step cascade spectra for each crystal multiplicity $m_{c r}$, which are the $\gamma$-ray energy spectra for fully detected cascades.

The ${ }^{234} U(n, \gamma)$ time-of-flight spectrum is shown in the left panel of Fig. 2. Besides the uranium resonances, a structure due to capture reactions in the Ti canning is observed above a few keV [6]. 

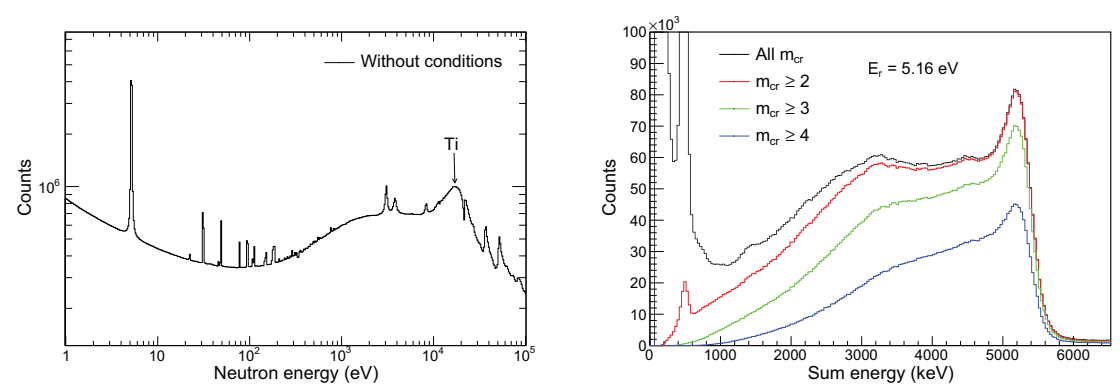

Figure 2. Time-of-flight spectrum converted to neutron energy (left). Total energy deposited for different sets of crystal multiplicities for ${ }^{234} \mathrm{U}(\mathrm{n}, \gamma)$ in the resonance at $5.16 \mathrm{eV}$ (right).

Fig. 2 (right) shows the sum-energy spectra in the resonance at $5.16 \mathrm{eV}$, corrected for background, for different multiplicity criteria [7]. All spectra clearly show the sum-peak at $5.3 \mathrm{MeV}$ corresponding to the $Q$ value of the reaction. There are differences between the spectra depending on the considered multiplicities. At low sum-energies (below $1 \mathrm{MeV}$ ) the spectrum for all $m_{c r}$ is dominated by the remaining background. However, in the spectra for $m_{c r} \geq 2$ this background is completely absent. For the present study, only cascades with $m_{c r} \geq 2$ are considered to ensure that the background is correctly subtracted, furthermore the multistep cascade spectra are constructed using only the events with sum-energy in the interval $4.8<E_{\text {sum }}(\mathrm{MeV})<6.0$.
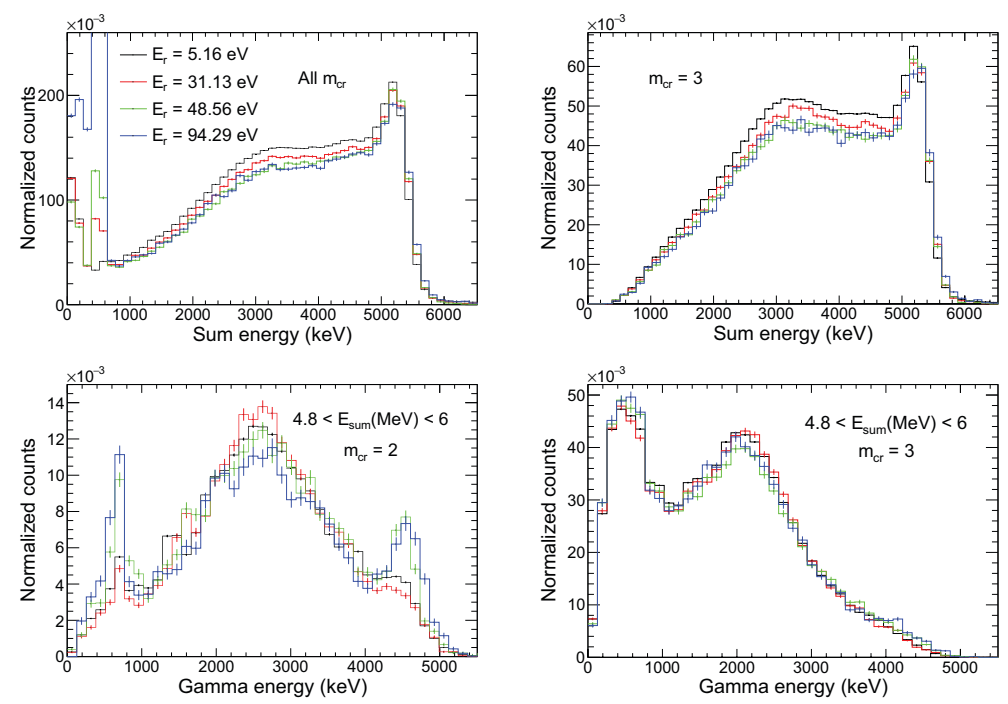

Figure 3. Sum-energy spectra of different resonances for all $m_{c r}$ and $m_{c r}=3$ (top). Multi-step $\gamma$-ray energy spectra of different resonances for $m_{c r}=2,3$ (bottom). The statistical uncertainties are small.

As can be seen in Fig. 3 (top), the sum-energy spectra for all $m_{c r}$ of four s-wave resonances show significant differences at low energy only. These differences are due to the fact that the subtraction of the background is approximative - the remaining background induced by the scattered neutrons is most apparent in the resonance at $E_{r}=94.29 \mathrm{eV}$ (blue) due to the 
larger scattering width. However, these differences do not appear for $m_{c r} \geq 3$ because of the low multiplicity nature of the background. More importantly, the background subtraction gets more accurate with increasing sum-energy, hence all sum-energy spectra have similar behavior for $E_{\text {sum }}>1 \mathrm{MeV}$.

The multi-step $\gamma$-ray energy spectra, see Fig. 3 (bottom), show similar responses for the different resonances for $m_{c r} \geq 3$. The spectra for $m_{c r}=2$ show significant differences depending on the considered resonance. These differences can be attributed to the Porter-Thomas fluctuations of primary transition intensities among the resonances, as expected the effects are mostly noticeable at the edges of the $m_{c r}=2$ multi-step $\gamma$-ray energy spectra.

The normalization of all spectra was done by dividing the spectra by the number of counts in the sum-energy spectrum of $m_{c r} \geq 2$ between $E_{\text {sum }}=4.8 \mathrm{MeV}$ and $6 \mathrm{MeV}$. The same normalization was applied to the simulations.

\section{Simulations}

The results presented in this work are based on the comparison of experimental data with statistical model simulations of $\gamma$ decay. The in-house developed Monte Carlo code DICEBoxc, based on the same algorithm used by F. Bečváŕ [8] in his code DicEBox, was used to simulate the gamma cascades while GEANT4 was used for the simulation of the interaction of these gammas with the complete TAC experimental assembly [2].

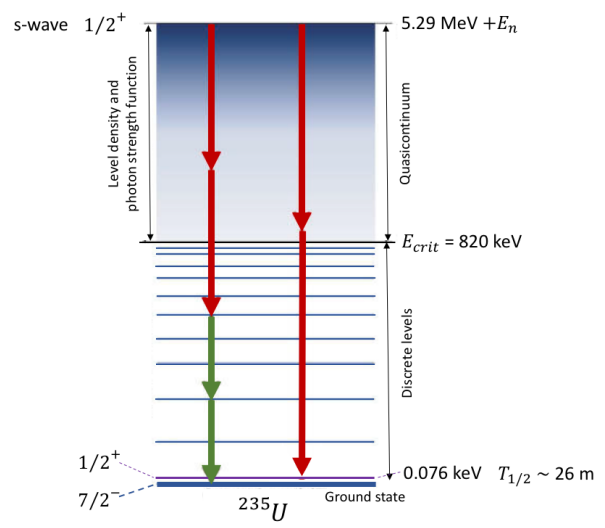

Figure 4. Schema of Monte Carlo cascades generation with Diceвоxc code. Red arrows depict the transitions generated in terms of LD and PSFs, green arows are the transitions among discrete levels taken from spectroscopic data.

DICEвоXс simulates sets of levels and their partial radiation widths known as nuclear realizations [8]. To describe the decay scheme, below a critical energy $E_{c r i t}$ all the level energies, spins, parities and branching intensities of depopulating transitions are taken from existing experimental data. Above $E_{c r i t}$, the level scheme is generated by the code - the levels are obtained by a random discretization of an a priori known LD formula. Further, the PSFs are used to generate probabilities of transitions of type $X$ (electric or magnetic) and multipolarity L. Fig. 4 shows a diagram of the operation of DIсевохc.

The partial radiation width of an electromagnetic transition from level $i$ to level $f, \Gamma_{i \gamma f}$, is selected from a Porter-Thomas distribution with the mean value $\left\langle\Gamma_{i \gamma f}^{X L}\right\rangle$ defined as

$$
\left\langle\Gamma_{i \gamma f}^{X L}\right\rangle=\frac{f^{X L}\left(E_{\gamma}\right) \cdot E_{\gamma}^{2 L+1}}{\rho\left(E_{i}, J_{i}, \pi_{i}\right)}
$$


where $\rho$ is the LD and $f^{X L}\left(E_{\gamma}\right)$ is the PSF. The $\gamma$-ray transition probabilities are corrected for internal conversion using tables from Ref. [9]. The levels and transitions below $E_{\text {crit }}$ are taken from ENSDF database [10]. To ensure satisfactory statistics concerning the modelled quantities, 20 nuclear realizations with $10^{5} \gamma$-cascades per realization were simulated for each combination of LD and PSFs models.

To simulate the transport and detection of $\gamma$-rays, the toolkit GEANT4 is used [11]. The geometry and efficiency of TAC have been accurately modeled following CAD drawings of the engineering design and direct measurements $[2,12]$. The modeled geometry is shown in the right panel of Fig. 1. Finally, an amplitude resolution of about 13-17\%, depending on the detector, and a threshold of $75 \mathrm{keV}$ for all crystals is assumed.

\subsection{Level density models}

The LD for given spin and parity is calculated as the product of three factors: the parity distribution $P(E, \pi)$, the spin distribution $R(E, J)$ and the $\operatorname{LD} \rho(E)$. In this work, one assumes that both parities are equally probable $P(E, \pi)=1 / 2$ at all $E$, while $R(E, J)$ is

$$
R(E, J)=\exp \left(-\frac{J^{2}}{2 \sigma_{c}^{2}}\right)-\exp \left(-\frac{(J+1)^{2}}{2 \sigma_{c}^{2}}\right) \approx \frac{2 J+1}{2 \sigma_{c}^{2}} \exp \left[-\frac{\left(J+\frac{1}{2}\right)^{2}}{2 \sigma_{c}^{2}}\right]
$$

where $\sigma_{c}$ is the spin cut-off factor. Different forms of spin cut-off factor could be used.

The DIceвoxc code includes various models for the $\operatorname{LD} \rho(E)$. The Constant Temperature (CT) [13] model assumes that the number of levels varies according to the constant temperature law and LD is given by

$$
\rho(E)=\frac{1}{T} \exp \left(\frac{E-E_{0}}{T}\right)
$$

with parameters $E_{0}$ and a nuclear temperature $T$, which are usually fitted to experimental discrete levels, taken from Ref. [14]. In this work the spin cut-off parameter, which is constant for a given nucleus [15], was used.

The Back-shifted Fermi Gas (BSFG) [16] model assumes the nucleus as a gas of fermions creating pairs and single particle levels are equally spaced and non-degenerated with a LD given by

$$
\rho(E)=\frac{\exp \left(2 \sqrt{a\left(E-E_{1}\right)}\right)}{12 \sqrt{2} \sigma_{c} a^{1 / 4}\left(E-E_{1}\right)^{5 / 4}},
$$

where $E_{1}$ is the energy backshift and $a$ is the LD parameter. The energy-dependent spin cut-off factor for the BSFG model was taken from Ref. [15]. The parameters for BSFG model were taken from Ref. [14]. Variations of the BSFG model have been developed, as for example in ref. [17], which incorporates the thermodynamic temperature $t$. In this case, the spin cut-off is related to a fraction of the moment of inertia of the nucleus that is usually taken between 0.5 and 1 . In addition, a BSFG model with energy-dependent LD parameter $a$ and spin cut-off which accounts for the damping of the shell effects was introduced in RIPL-3 [18]. Finally, we used microscopic LD in the form of numerical interpolation tables calculated with the Hartree-Fock-Bogoliubov (HFB) method [19, 20].

\subsection{Photon strength function}

The statistical decay of compound nuclei from excitation energies above neutron separation energy is dominated by the $E 1$ transitions due to the presence of the giant dipole electric 
resonance (GDER). The shape of the $E 1$ PSF for deformed nucleus is usually described by a sum of two standard Lorentzians [18, 21], as a consequence of vibration modes along and perpendicular to the symmetry axis. This description is known as Standard Lorentzian model (SLO):

$$
f_{S L O}^{E 1}\left(E_{\gamma}\right)=\frac{1}{3(\pi \hbar c)^{2}} \sum_{i=1}^{2} \frac{\sigma_{G_{i}} E_{\gamma} \Gamma_{G_{i}}^{2}}{\left(E_{\gamma}^{2}-E_{G_{i}}^{2}\right)^{2}+E_{\gamma}^{2} \Gamma_{G_{i}}^{2}},
$$

where the parameters $E_{G_{i}}, \Gamma_{G_{i}}$ and $\sigma_{G_{i}}$ are the energy, width and cross section of the GDER.

Different variations were proposed to better describe the energy region below neutron separation energy. The model by Kadmenskii, Markushev and Furman (KMF) [22] aims only at this energy region while generalised Lorentzian models by Kopecky, Uhl and Chrien (GLO, ELO, EGLO) [23] and other models and calculations attempt to describe the E1 PSF in the whole energy region.

The KMF, GLO and ELO models use the damping width $\Gamma_{T}\left(E_{\gamma}, T_{f}\right)$ which depends on $E_{\gamma}$ and the nuclear temperature $T_{f}$ in form

$$
\Gamma_{T}\left(E_{\gamma}, T_{f}\right)=\frac{\Gamma_{G}}{E_{G}^{2}}\left(E_{\gamma}^{2}+4 \pi^{2} T_{f}^{2}\right) .
$$

Phenomenological modifications of this damping width in which is introduced a $k$ parameter were proposed in the EGLO [23] and the MGLO [24] models. There are other models for $E 1$ PSF, we refer the reader to the overview in RIPL-3 [18].

For the decay of levels below the neutron separation energy, $M 1$ transitions play an important role. In this work the $M 1$ PSF consists of the spin-flip (SF) resonance, which dominates the $M 1 \mathrm{PSF}$ at relatively high energy typically around $7 \mathrm{MeV}$, and the scissor mode (SC), a concentration of $M 1$ strength around 2-3 MeV in deformed nuclei. The SLO model was adopted to describe the M1 PSF. For further details see review [25] and references therein.

The electric quadrupole (E2) transitions, although playing a minor role with respect to dipole transitions, are also taken into account. The SLO model with a single Lorentzian was used to describe the E2 PSF as recommended in [18].

\section{Comparison of simulations and measurements}

Various combinations of LD and PSF models were checked and compared with the experimental data introduced in Sec. 2. The parameters were taken from RIPL-3 database [18] in which only one SLO term for the M1 PSF is recommended, or from original works, (i) the analysis of $\mathrm{d}$ - and ${ }^{3} \mathrm{He}$-induced reactions on actinide targets performed at the Oslo Cyclotron Laboratory (OCL) [26] and (ii) the measurement of multi-step $\gamma$-ray energy spectra from resonant neutron capture on uranium samples with DANCE calorimeter [27]. In both a sum of SLO terms was adopted to describe the M1 PSF - one for the SF and two for the SC. The $E 2$ transitions were included in the simulations by taking the parameters from [28]. The parameters use for the PSF in the different simulations are collected in Table. 1.

From the E1 PSF models introduced in Sec. 3.2 the SLO and KMF models do not reproduce the experimental data in combination with any LD model independently on the chosen parametrisation of the M1 PSF. Conversely, the ELO, GLO, EGLO and MGLO E1 PSF models, paired with a suitable LD model, allow, by tuning the parameters of the M1 PSF and the $k$ parameter, a satisfactory description of the experimental data.

In Fig. 5 we compare experimental data with simulations using the PSFs parameters taken from (i) the RIPL-3 database [18] with GLO for $E 1$ and SLO for $M 1$ PSF, (ii) the DANCE 
analysis [27] and (iii) the analysis of $\mathrm{d}-$ and ${ }^{3} \mathrm{He}$-induced reactions at OCL [26]. The standard deviation due to different nuclear realizations is only calculated in the simulation for RIPL-3, for the other simulations the standard deviation shows similar behaviour and is not displayed for a better visualization. The statistical uncertainties are much smaller than the standard deviation. Overall, the introduction of the SC is mandatory for the improvement of the simulation and the increase of the SC strength in the DANCE analysis with respect to OCL improves the description of the experimental data. It may be possible that in order to match experimental data the SC strength has to be further increased and used in conjunction with steeper $E 1$ PSF of generalised Lorentzian type.
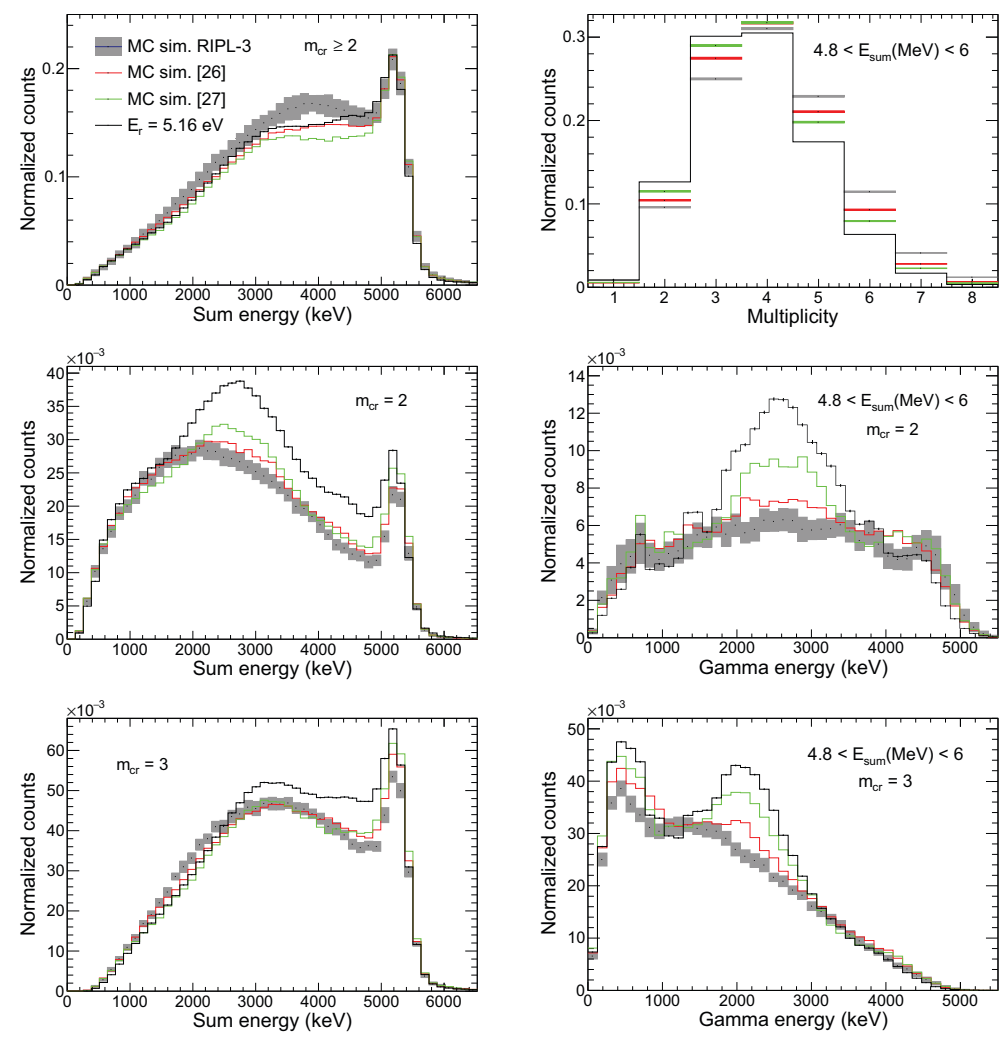

Figure 5. Comparison of experimental data to simulations using the LD and PSF models as recommended in RIPL-3 database [18] (grey filled), and as published in Refs. [26] (red line) and [27] (green line). The left column shows the total deposited energy spectra while on the right the multiplicity distribution and multi-step $\gamma$-ray energy spectra are shown. The resonance energy as well as the multiplicity and sum-energy conditions are specified in the figures.

\section{Conclusion}

The Total Absorption Calorimeter at the n_TOF facility (CERN) was used to measure the $\gamma$-ray cascades following the neutron capture in ${ }^{234} \mathrm{U}$. Simulations of $\gamma$ decay performed with DICEвоXc for various LD and PSFs combinations were compared with the experimental data. The inadequacy of the SLO and KMF models of E1 PSF as well as the necessity of scissors mode contribution to M1 PSF was shown. The simulations with model combinations proposed in OCL and DANCE analyses do not reproduce our data. This analysis will continue 
with a detailed parameter search for analytical models and the use of tabulated PSFs from QRPA calculations [29], as well as the extension to other actinides.

Table 1. Parameters from RIPL-3 [18], OCL [26] and DANCE [27] for the PSFs.

\begin{tabular}{cccccccccc}
\hline Transition & $E_{1}(\mathrm{MeV})$ & $\Gamma_{1}(\mathrm{MeV})$ & $\sigma_{1}(\mathrm{mb})$ & $E_{2}(\mathrm{MeV})$ & $\Gamma_{2}(\mathrm{MeV})$ & $\sigma_{2}(\mathrm{mb})$ & $E_{3}(\mathrm{MeV})$ & $\Gamma_{3}(\mathrm{MeV})$ & $\sigma_{3}(\mathrm{mb})$ \\
\hline E1 [18] & 11.11 & 1.12 & 243.3 & 13.41 & 4.98 & 426 & - & - & - \\
M1 [18] & - & - & - & - & - & - & 6.61 & 4.00 & 2.35 \\
E2 [28] & 10.21 & 1.18 & 1.7 & - & - & - & - & - & - \\
\hline E1 [26] & 11.40 & 4.20 & 572 & 14.40 & 4.20 & 1040 & 7.30 & 2.0 & 15.0 \\
M1 [26] & 2.15 & 0.80 & 0.45 & 2.90 & 0.60 & 0.40 & 6.61 & 4.00 & 7.00 \\
E2 [28] & 10.21 & 1.18 & 1.7 & - & - & - & - & - & - \\
\hline E1 [27] & 11.28 & 2.48 & 325 & 13.73 & 4.25 & 384 & & & \\
M1 [27] & 2.15 & 0.80 & 0.60 & 2.90 & 0.60 & 0.53 & 6.61 & 4.00 & 1.50 \\
E2 [28] & 10.21 & 1.18 & 1.7 & - & - & - & - & - & - \\
\hline
\end{tabular}

\section{References}

[1] W. Hauser, H. Feshbach, Phys. Rev. 87, 366 (1952)

[2] C. Guerrero et al. (n_TOF), Nucl. Instrum. Meth. A 608, 424 (2009)

[3] E. Chiaveri et al. (n_TOF), Nucl. Data Sheets 119, 1 (2014)

[4] C. Guerrero et al. (n_TOF), Eur. Phys. J. A 49, 27 (2013)

[5] W. Dridi, PhD thesis (Université d'Evry Val d'Essonne, 2006)

[6] C. Lampoudis et al. (n_TOF), in Proceedings of the 2007 International Conference on Nuclear Data for Science and Technology (2008), Vol. 1, pp. 595-598

[7] W. Dridi et al. (n_TOF), in Conference Proceedings of the American Nuclear Society's Topical Meeting on Reactor Physics (2006), Vol. C032, pp. 1-10

[8] F. Bečvář, Nucl. Instrum. Meth. A 417, 434 (1998)

[9] R.S. Hager, E.C. Seltzer, Nucl. Data A4, 1 (1968)

[10] E. Browne, J. Tuli, Nucl. Data Sheets 122, 205 (2014)

[11] S. Agostinelli et al. (GEANT4), Nucl. Instrum. Meth. A 506, 250 (2003)

[12] C. Guerrero et al. (n_TOF), Nucl. Instrum. Meth. A 671, 108 (2012)

[13] A. Gilbert, A.G.W. Cameron, Can. J. Phys. 43, 1446 (1965)

[14] T. von Egidy, D. Bucurescu, Phys. Rev. C 72, 044311 (2005)

[15] T. von Egidy, H.H. Schmidt, A.N. Behkami, Nucl. Phys. A 481, 189 (1988)

[16] H.A. Bethe, Phys. Rev. 50, 332 (1936)

[17] W. Dilg, W. Schantl, H. Vonach, M. Uhl, Nucl. Phys. A 217, 269 (1973)

[18] R. Capote et al., Nucl. Data Sheets 110, 3107 (2009)

[19] S. Goriely, S. Hilaire, A.J. Koning, Phys. Rev. C 78, 064307 (2008)

[20] A.J. Koning, S. Hilaire, S. Goriely, Nucl. Phys. A 810, 13 (2008)

[21] G.A. Bartholomew, Annual Review of Nuclear Science 11, 259 (1961)

[22] S.G. Kadmenskii, V.P. Markushev, V.I. Furman, Sov. J. Nucl. Phys. 37, 165 (1983)

[23] J. Kopecky, M. Uhl, R.E. Chrien, Phys. Rev. C 47, 312 (1993)

[24] J. Kroll et al., Phys. Rev. C 88, 034317 (2013)

[25] K. Heyde, P. von Neumann-Cosel, A. Richter, Rev. Mod. Phys. 82, 2365 (2010)

[26] M. Guttormsen et al., Phys. Rev. C 89, 014302 (2014)

[27] J.L. Ullmann et al., Phys. Rev. C 96, 024627 (2017)

[28] Handbook for Calculations of Nuclear Reaction Data Reference Input Parameter Library, IAEA-TECDOC-1034 (Vienna, 1998)

[29] S. Goriely et al., Phys. Rev. C 98, 014327 (2018) 